ORIGINAL ARTICLE

\title{
Heterotaxia syndrome: the role of screening for intestinal rotation abnormalities
}

\author{
M Choi, S H Borenstein, L Hornberger, J C Langer

See end of article for authors' affiliations ......................

Correspondence to: Dr J C Langer, Division of General Surgery, Hospital for Sick Children, 555 University Avenue, Toronto, Ontario M5G 1X8, Canada; jacob. langer@sickkids.ca

Accepted 2 April 2005

Published Online First 12 May 2005
Background: Heterotaxia syndrome involves multiple anomalies, including cardiac malformations and intestinal rotation abnormalities. Most authors recommend routine radiological evaluation, with laparotomy and Ladd procedure if a rotation abnormality is found.

Aims: To determine if routine radiological screening is necessary, and if there is a group of children that can safely be managed expectantly.

Methods: Retrospective chart review of all children with heterotaxia syndrome from 1968 to 2002.

Results: Complete data were available for 177 patients. Twenty five (14\%) had neonatal gastrointestinal symptoms (feeding intolerance, vomiting). Eleven of these had gastrointestinal contrast studies, of which seven were abnormal and led to surgery. Of the 152 asymptomatic neonates, nine had radiological screening and six of these were abnormal. Only one was thought to have a narrow based mesentery, but did not undergo surgery due to cardiac disease. There were no intestinal complications on follow up in this group. The other 143 asymptomatic children did not undergo radiological screening and were closely followed. Four subsequently developed gastrointestinal symptoms and had contrast studies; only one of these had malrotation and underwent a Ladd procedure. Of the remaining 139 patients who remained asymptomatic, $60(43 \%)$ died of cardiac disease and none developed intestinal symptoms or complications related to malrotation on follow up.

Conclusion: Asymptomatic children with heterotaxia syndrome have a low risk of adverse outcome related to intestinal rotation abnormalities. Routine screening may not be necessary as long as close follow up is done, and prompt investigation is performed for those that develop gastrointestinal symptomatology.
1 testinal rotational abnormalities (IRA) are frequently found in patients with heterotaxia syndrome, which is defined as any arrangement of organs along the left-right body axis that differs from complete situs solitus or complete situs inversus. ${ }^{1-3}$ The most clinically relevant IRA is malrotation, which because of the presence of a narrow based mesentery can predispose to midgut volvulus and intestinal ischaemia. Heterotaxia is usually associated with polysplenia or asplenia, and because cardiac development is also dependent on the formation of normal right-left orientation, congenital heart disease is usually present as well. Advances in cardiac surgery have prolonged the survival of patients with heterotaxia syndrome, and therefore managing the associated intra-abdominal anomalies has become part of their overall care. ${ }^{45}$

The consensus in the literature is that children with heterotaxia should be screened with upper gastrointestinal contrast studies to detect rotation abnormalities that may predispose to volvulus. If such an abnormality is found, a Ladd procedure is usually recommended. ${ }^{6-8}$ However, recent publications have challenged the necessity for operation in every patient with an intestinal rotation abnormality. ${ }^{9}$ These studies raise the possibility that the natural history of asymptomatic intestinal rotation abnormalities may be relatively benign, and suggest that there may be a role for an expectant approach in patients with heterotaxia syndrome. Based on these considerations, we have adopted a more conservative approach to the management of these patients. Recommendations for surgical intervention are based on a combination of clinical symptoms and gastrointestinal imaging. In this paper, we report on our experience with this conservative, individualised approach to IRA in patents with heterotaxia.

\section{METHODS}

Approval for this study was obtained from the Research Ethics Board at the Hospital for Sick Children. Patients with heterotaxia syndrome with and without structural heart disease diagnosed between 1968 and 2002 were identified through our cardiology database and medical records department. The clinical course of each child was reviewed retrospectively focusing on the development of symptoms of intestinal obstruction, the results of gastrointestinal imaging studies, whether or not the child required surgical intervention and the length of clinical follow up.

While there is some discrepancy in the literature with respect to how one defines various forms of IRA, for the purpose of this study we used the following definitions. In classic malrotation, the duodenum and caecum overlap and the superior mesenteric vessels are contained within a narrow mesentery which is predisposed to volvulus. ${ }^{11}$ There is universal agreement that these patients require urgent Ladd procedure. ${ }^{12-14}$ In non-rotation, the small bowel is on the right, the colon is on the left, and the base of the mesentery is wide. These patients are not at risk for midgut volvulus, and in fact the goal of the Ladd procedure is to put the bowel into this position. Between these two rotational states are varying degrees of intestinal rotation, which depending on the length of the mesentery may or may not predispose to midgut volvulus.

\section{RESULTS}

Two hundred and twenty one children diagnosed with heterotaxia were identified through our database. Complete records were available on 177 patients, who comprised our study group. Twenty five patients (14\%) developed gastrointestinal symptoms within eight weeks of birth (table 1). 
Eleven of these 25 patients were investigated with gastrointestinal contrast studies: one was normal, one revealed a hiatal hernia, two showed what was thought to be nonpathological IRA, and seven were thought to show malrotation. Six of the latter seven children underwent emergency laparotomy and one did not due to cardiac instability and subsequent cardiac failure. Of the six patients who underwent laparotomy, four were found to have malrotation and two were found to have duodenal web. One of the patients with malrotation developed necrotising enterocolitis several weeks postoperatively and required extensive bowel resection. This child ultimately died at 1 year of age from complications secondary to short bowel syndrome. One neonate had severe gastro-oesophageal reflux on upper gastrointestinal imaging which eventually required fundoplication. None of the 14 symptomatic neonates who were not imaged developed intestinal complications at a median follow up of 3 weeks (range 0-96 months).

Of the remaining 152 asymptomatic heterotaxia patients, nine had radiological screening studies. Three studies were normal. One child with severe congenital heart disease was suspected of having a jejunal obstruction, but her cardiac status precluded further investigation and surgical intervention. In the other five screened patients, the studies showed various forms of IRA, but none were thought to have narrow based mesenteries and none underwent surgery. There were no intestinal complications in this group on median follow up of 18 months (range 4-216 months).

The other 143 asymptomatic children did not undergo radiological screening and were closely followed. Four (3\%) eventually developed gastrointestinal symptoms (table 1) which prompted radiological investigation. One study was normal. Two studies revealed IRA with what was thought to be a broad based mesentery that did not require surgery. The fourth child presented when he was 16 years old with recurrent abdominal pain and was found to have situs inversus with malrotation and a narrow based small bowel mesentery. At laparotomy, the bowel was not ischaemic and a Ladd procedure was performed. Of the remaining 139 patients who remained asymptomatic, 60 (43\%) died of cardiac disease and none developed symptoms of intestinal obstruction or ischemia at median follow up of 114 months (range 0.1-252 months).

\section{DISCUSSION}

Following its extension into the umbilical cord during the tenth week of gestation, the intestine normally rotates 270 degrees around the axis of the superior mesenteric artery (SMA) as it migrates back into the abdominal cavity. This rotation brings the duodenojejunal flexure under the SMA and fixes it to the retroperitoneum of the left upper quadrant.

\begin{tabular}{lcc|}
$\begin{array}{l}\text { Table } 1 \text { Gastrointestinal symptoms among heterotaxia } \\
\text { patients }\end{array}$ & No. & $(\%)$ \\
\hline & 12 & $(48)$ \\
\hline Neonates $(\mathbf{n}=25)$ & 6 & $(24)$ \\
Feeding intolerance & 5 & $(20)$ \\
Abdominal distension & 3 & $(12)$ \\
Bilious vomiting & 2 & $(8)$ \\
Non-bilious vomiting & 2 & $(8)$ \\
Delayed passage of meconium & & \\
Lower gastrointestinal bleed & 3 & $(75)$ \\
& 2 & $(50)$ \\
Non-neonates ( $\mathbf{n}=4)$ & 2 & $(50)$ \\
Non-bilious vomiting & & \\
Abdominal pain & & \\
Abdominal distension & &
\end{tabular}

Similarly, the cecocolic limb rotates into the right lower quadrant. Following fusion of the mesentery with the peritoneum, the small intestines become attached to the posterior abdominal wall by a broad based mesentery, which contains the SMA and its branches. This rotation ensures that the SMA is distributed along a broad mesenteric base which minimises the possibility of postnatal midgut volvulus. In malrotation, the caecum and duodenojejunal loop remain in the mid upper abdomen. The mesenteric attachment of the small intestine is therefore very narrow and predisposed to potentially fatal torsion with necrosis of the entire midgut. In non-rotation, no additional rotation occurs following migration of the intestines into the abdominal cavity; the small bowel remains on the right side of the abdomen while the colon lies on the left. Between these two extremes is a spectrum of rotational abnormalities in which partial rotation of the intestine around the SMA occurs. Surgeons have traditionally operated on all patients with IRA because there are no clear criteria defining which of these rotational abnormalities predispose to volvulus.

In our hospital, patients with heterotaxia are not routinely screened for IRA. Most of these patients are managed conservatively with close clinical follow up. If, as the literature suggests, ${ }^{6-8}$ these patients are genuinely predisposed to midgut volvulus, we would have expected a significant proportion of heterotaxia patients to present emergently with signs and symptoms of intestinal obstruction and/or ischaemia. However, our data suggest that this was not the case. Of our 177 heterotaxia patients, only five had symptomatic classic malrotation, four of whom presented with symptoms during the neonatal period, and none of whom developed intestinal ischaemia or bowel loss due to midgut volvulus. The one late death among these five children was due to necrotising enterocolitis rather than midgut volvulus.

There were a significant number of symptomatic children in our series who were not screened. Most of these children had very severe forms of heterotaxia syndrome, usually involving severe congenital cardiac anomalies. Because it was clear that they would not survive beyond the neonatal period and would never be candidates for surgery, these neonates were treated palliatively. The other children in this group had mild symptoms that were not thought at the time to be consistent with an IRA. Because of the relatively high incidence of IRA in the symptomatic group that were screened, and the potentially serious consequences of missing a child with classic malrotation, we would no longer withhold radiological screening studies from these children, and currently recommend radiological screening studies for every symptomatic child with heterotaxia, unless it is clear that the patient's condition is incompatible with survival beyond the neonatal period.

There were a number of children in our series who had radiological screening studies that showed an IRA which was thought to be associated with a wide enough mesenteric base to prevent midgut volvulus ("non-pathological IRA"). Although many surgeons have operated routinely on any child with an IRA, we have felt that this group could be managed expectantly. Our data support this view, since none of these children have developed intestinal obstruction or ischaemia during follow up. This approach is also supported by a recent study by Mehall et al who concluded that not all patients require surgery for IRA. ${ }^{10}$ Among their 101 patients with "atypical malrotation", defined as an abnormality of intestinal rotation somewhere along the spectrum between malrotation and non-rotation, only two developed volvulus, which was non-ischaemic in both cases.

The 139 asymptomatic children with heterotaxia who have not been subjected to gastrointestinal contrast studies have not developed complications related to malrotation after a 


\section{What is already known on this topic}

- Heterotaxia syndrome is often associated with intestinal rotation abnormalities

- Some series of heterotaxia syndrome have included cases of malrotation and volvulus, and the authors have therefore suggested an aggressive programme of investigation and treatment for rotation abnormalities in every case of heterotaxia syndrome

- Many intestinal rotation abnormalities do not predispose the child to volvulus, and therefore do not require surgical treatment

median of 9 years of follow up. This supports our practice of reserving screening radiological studies only for symptomatic patients. In a comparison of risks, the $0.6 \%$ incidence of volvulus in our population is much lower than the $15 \%$ risk of adhesive small bowel obstruction following laparotomy for malrotation ${ }^{15}$ which is quoted in the literature.

We recommend an individualised approach to the management of children with heterotaxia. Asymptomatic patients can be followed as long as compliance with regular follow up is certain. When follow up is uncertain or when gastrointestinal symptoms develop, prompt radiological investigation is required. If investigation reveals classic malrotation, the child should undergo a Ladd procedure, and if investigation reveals normal rotation or non-rotation the child can be safely observed. When the risk of midgut volvulus in symptomatic patients is unclear from the radiological studies, laparoscopy ${ }^{16}$ or laparotomy should be done, with subsequent Ladd procedure for those in whom the mesenteric base is felt to be narrow. ${ }^{17}$

\section{Authors' affiliations}

M Choi, S H Borenstein, L Hornberger, J C Langer, Divisions of General Surgery and Cardiology, The Hospital for Sick Children, University of Toronto, Toronto, Ontario, Canada

Competing interests: none declared

\section{REFERENCES}

1 Zlotogora J, Elian E. Asplenia and polysplenia syndromes with abnormalities of lateralisation in a sibship. J Med Genet 1981;18:301-2.

\section{What this study adds}

- No previous study of this population has taken a conservative approach to the investigation and treatment of intestinal rotation abnormalities

- We have shown the safety of a conservative approach, as long as children are followed closely and investigated if they become symptomatic

- A conservative approach results in better use of resources and minimises radiation in these children, and should therefore be considered

2 Mishalany H, Mahnovski V, Woolley M. Congenital asplenia and anomalies of the gastrointestinal tract. Surgery 1982;91:38-41.

3 Ruben GD, Templeton JM Jr, Ziegler MM. Situs inversus: the complex inducing neonatal intestinal obstruction. J Pediatr Surg 1983;18:751-6.

4 Albanese SB, Carotti A, Di Donato RM, et al. Bidirectional cavopulmonary anastomosis in patients under two years of age. J Thorac Cardiovasc Surg 1992; 104:904-9.

5 Cetta F, Feldt RH, O'Leary PW, et al. Improved early morbidity and mortality after Fontan operation: the Mayo Clinic experience, 1987 to 1992. J Am Coll Cardiol 1996;28:480-6.

6 Chang J, Brueckner M, Touloukian RJ. Intestinal rotation and fixation abnormalities in heterotaxia: early detection and management. J Pediatr Surg 1993;28:1281-4.

7 Nakada K, Kawaguchi F, Wakisaka M, et al. Digestive tract disorders associated with asplenia/polysplenia syndrome. J Pediatr Surg 1997;32:91-4.

8 Ditchfield MR, Hutson JM. Intestinal rotational abnormalities in polysplenia and asplenia syndromes. Pediatr Radiol 1998;28:303-6.

9 Dilley AV, Pereira J, Shi EC, et al. The radiologist says malrotation: does the surgeon operate? Pediatr Surg Int 2000;16:45-9.

10 Mehall JR, Chandler JC, Mehall RL, et al. Management of typical and atypical intestinal malrotation. J Pediatr Surg 2002;37:1169-72.

11 Ladd WE. Congenital obstruction of the duodenum in children. N Engl J Med 1932;206:277-83.

12 Powell DM, Othersen HB, Smith CD. Malrotation of the intestines in children: the effect of age on presentation and therapy. J Pediatr Surg 1989;24:777-80.

13 Rescorla FJ, Shedd FJ, Grosfeld JL, et al. Anomalies of intestinal rotation in childhood: analysis of 447 cases. Surgery 1990;108:710-15.

14 Torres AM, Ziegler MM. Malrotation of the intestine. World J Surg 1993;17:326-31.

15 Wilkins BM, Spitz L. Incidence of postoperative adhesion obstruction following neonatal laparotomy. Br J Surg 1986;73:762-4.

16 Mazziotti MV, Strasberg SM, Langer JC. Intestinal rotation abnormalities without volvulus: the role of laparoscopy. J Am Coll Surg 1997; 185:172-6.

17 Chen LE, Minkes RM, Langer JC. Laparoscopic vs open surgery for malrotation without volvulus. Pediatr Endosurg Innov Tech 2003;7:433-8. 